\title{
Self-perception of smile attractiveness as a reliable predictor of increased patient compliance with an orthodontist
}

\author{
Michał Sarul ${ }^{1, A-F}$, Joanna Antoszewska-Smith ${ }^{1, A, C, E, F}$, Hyo-Sang Park ${ }^{1,2, E, F}$ \\ ${ }^{1}$ Department of Dentofacial Orthopedics and Orthodontics, Wroclaw Medical University, Poland \\ ${ }^{2}$ Department of Orthodontics, Kyungpook National University, Daegu, South Korea \\ A - research concept and design; $B$ - collection and/or assembly of data; $C$ - data analysis and interpretation; \\ $D$ - writing the article; $E$ - critical revision of the article; $F$ - final approval of the article
}

\section{Address for correspondence \\ Michał Sarul}

E-mail:michal.sarul@gmail.com

Funding sources

None declared

Conflict of interest

None declared

Received on 0ctober 10, 2018

Reviewed on November 20, 2018

Accepted on June 27, 2019

Published online on November 28, 2019

Cite as

Sarul M, Antoszewska-Smith J, Park H-S. Self-perception of smile attractiveness as a reliable predictor of increased patient compliance with an orthodontist. Adv Clin Exp Med. 2019;28(12):1633-1638. doi:10.17219/acem/110320

DOI

10.17219/acem/110320

\section{Copyright}

Copyright by Author(s)

This is an article distributed under the terms of the

Creative Commons Attribution 3.0 Unported (CC BY 3.0)

(https://creativecommons.org/licenses/by/3.0/)

\begin{abstract}
Background. Predicting the cooperation of orthodontic patients seems to be of the utmost importance for successful results in treatment with removable appliances, especially if their cost is to be covered from public funding. Therefore, the issue of unbiased pre-treatment assessment of cooperation still calls for an investigation.
\end{abstract}

Objectives. The objective of this study was to check whether smile attractiveness and its importance, subjectively evaluated by the patients/their caregivers, are reliable predictors of a patient's compliance during treatment with removable appliances.

Material and methods. The study group comprised 97 patients aged 9-12 years, treated with active plates or twin-blocks, equipped with the TheraMon ${ }^{\circledR}$ system. Before treatment, the caregivers and the patients filled out the questionnaires ranking both of the investigated variables. After 9 months of treatment, we analyzed the correlations of the daily wear time (DWT) and other variables.

Results. The analysis revealed the following: no relevance of the DWT to the type of removable appliances; negative correlation between the DWT and smile attractiveness assessed by the children and their caregivers; evidently elongated DWT (up to $9.68 \mathrm{~h}$ in children), who, together with the caregivers, evaluated their smile attractiveness as poor; coherence of children's and their caregivers' responses evaluating smile attractiveness and its importance, as well as the lack of consistency when comparing responses provided separately by the children and their caregivers.

Conclusions. Application of the TheraMon ${ }^{\circledR}$ sensors objectively proved that the patient's smile attractiveness ranked subjectively as low predicts the orthodontic patient's irreproachable cooperation. Therefore, our easy-to-use questionnaire calls for changing the protocol regarding the wearing time of removable appliances during treatment and introducing an evidence-based policy of reimbursement for such therapy from public funds. Further investigation of the effectiveness of removable appliances worn shorter than previously presumed and of the motivation to continue treatment, once smile attractiveness has been improved, is necessary.

Key words: self-perception of smile attractiveness, orthodontic appliances, cooperative behavior 


\section{Introduction}

Nowadays, the alarming prevalence of malocclusions, which affect more than $90 \%$ of the population, ${ }^{1,2}$ calls for interceptive orthodontic treatment, which improves the future general health status. Such early intervention is mostly realized with removable appliances, whose success is largely dependent on patient cooperation. ${ }^{3-5}$ Monitoring patient cooperation has just recently been revolutionized due to microsensors, e.g., the TheraMon ${ }^{\circledR}$ system. These microsensors are almost imperceptible; they are imbedded in an acrylic plate, which does not affect comfort while wearing the orthodontic device. TheraMon ${ }^{\circledR}$ is also resistant to any interference from the patient, thus measuring wearing time accurately and reliably. ${ }^{6,7}$

Regrettably, studies using the electronic chips efficiently demonstrated considerably weaker patient cooperation than previously thought. ${ }^{8}$ Therefore, many researchers focused on establishing factors that would enable us to predict patient cooperation before manufacturing an appliance. ${ }^{9-12}$ Our previous studies ${ }^{13}$ similarly to those by Amado et al., ${ }^{14}$ have shown that the degree of compliance depends on the patients' and - more significantly - their caregivers' personality traits.

Such findings automatically gave rise to a question: is it possible to omit a complex psychological questionnaire and still predict cooperation reliably, especially that early orthodontic treatment is a part of the dental prophylaxis, which in many countries is reimbursed from public funds?

Bearing in mind that impaired smile attractiveness has been the main incentive to seek orthodontic treatment, ${ }^{15-18}$ one might have presumed that the individuals who are dissatisfied with their smiles are likely to be the most compliant patients. However, since eager amenability to the regimen of treatment with removable appliances is an uncertain issue, and a strong relationship between poor smile esthetics and excellent patient cooperation has not yet been objectively verified, our hypothesis obviously remains a matter of controversy and debate.

Therefore, the purpose of our study was to provide evidence whether objectively, electronically monitored daily wear time (DWT) of removable devices correlates with selfperception of smile attractiveness assessed by patients and their caregivers. If so, we would have established an easy and reliable predictor of compliance, thus decreasing the orthodontic treatment cost/effectiveness ratio.

\section{Material and methods}

\section{Study group}

Ninety-seven healthy children ( 51 girls, 46 boys) without cleft palate or any oral disease, and qualified for interceptive or early orthodontic treatment with removable appliances, were enrolled in the study that received approval from the Ethical Review Committee of Wroclaw Medical University (approval No. KB-322/2014).

All patients met the following inclusion criteria: age 9-12 years, good health and no syndromes or clefts, malocclusion likely to improve with an active plate or twin block, i.e., mild or moderate crowding of the anterior maxillary teeth, Class II functional shift, or pseudo Class III or bilateral cross-bite due to narrowed maxillary dental arch.

Before treatment, the caregivers ( 79 females, 18 males), who declared future active supervision of the child's cooperation, signed the informed consent. Subsequently, all the caregivers and the patients responded to the questionnaires designed by a psychologist qualified in assessing pro-health behavior. The following factors were ranked:

- A1: children's self perception of smile attractiveness,

- A2: children's thoughts on the importance of smile attractiveness,

- B1: caregivers' perception of their children's smile attractiveness,

- B2: caregiver's thoughts on the importance of smile attractiveness.

Regarding the attractiveness of the patient's smile, the score was given from 1 to 5 as very poor, poor, fair, great, and excellent, respectively. Regarding the importance of smile attractiveness itself, the score was given from 1 to 5 as very indifferent, indifferent, barely important, important, and very important, respectively.

In each case, a psychologist participating in these studies explained to the patients are caregivers all the terms appearing in the form, which was completed only after the terminology of the questionnaire was understandable for the respondent.

Subsequently, 56 children ( 29 girls, 27 boys) received a twin block and 41 children (22 girls, 19 boys) received an active plate, all devices equipped with the TheraMon ${ }^{\circledR}$ electronic sensors of temperature fluctuation, measuring appliance wear time with an accuracy of $15 \mathrm{~min}$. We asked the patients to wear their appliances at least 12-14 h per day and to report back every 6 weeks on the DWT measurements with the TheraMon ${ }^{\circledR}$ software. At that time, all patients appeared on designated appointments and none of the patients were excluded from the study.

In order to assess the accuracy of the TheraMon ${ }^{\circledR}$ sensors in vivo, prior to this study we applied the protocol described by Pauls et al. ${ }^{19}$ To do so, we instructed 5 postgraduate students from our Department to wear Schwarz plates equipped with the TheraMon ${ }^{\circledR}$ sensors for 1 week, reading records provided by the sensors every day and noting every hour the device insertion and removal. The data analysis revealed insignificant mean discrepancy of $9.6 \mathrm{~min}$ per day between the recorded and the actual DWTs.

After 9 months, we subjected all the gathered data to a statistical analysis to answer the following research questions: 
1. Is the DWT indifferent due to type of the removable device used in our study?

2. Does any of the 4 variables (A1-B2) affect the DWT and if yes, then how?

3 . Is there any correlation between the 4 variables facilitating psychological evaluation process?

\section{Statistical analysis}

STATISTICA v. 12 software (StatSoft, Inc., Tulsa, USA) enabled us to answer our queries. The Shapiro-Wilk normality test results proved that the range of our data fell beyond the normal distribution pattern, so we applied a non-parametric analysis. All data was processed using the Mann-Whitney U test, Spearman's and Kendall's т rank correlation coefficients and Dunn's multiple comparison test. ${ }^{20-22}$ Power test analysis was performed with the Student's t-test, evaluating the significance of Spearman's R correlation coefficient. We established the significance level at $\mathrm{p}<0.05$ and the threshold of the power test analysis at the level of 0.95 .

\section{Results}

Measurements of the daily wear time did not vary in the patients wearing twin block or an active plate (Table 1).

Figure 1 demonstrates percentage distributions of the ranks obtained in the questionnaires. As for statistics, all 4 variables significantly $(\mathrm{p}<0.05)$ correlated with the DWT: A2 and B2 - positively, A1 and B1 - negatively.
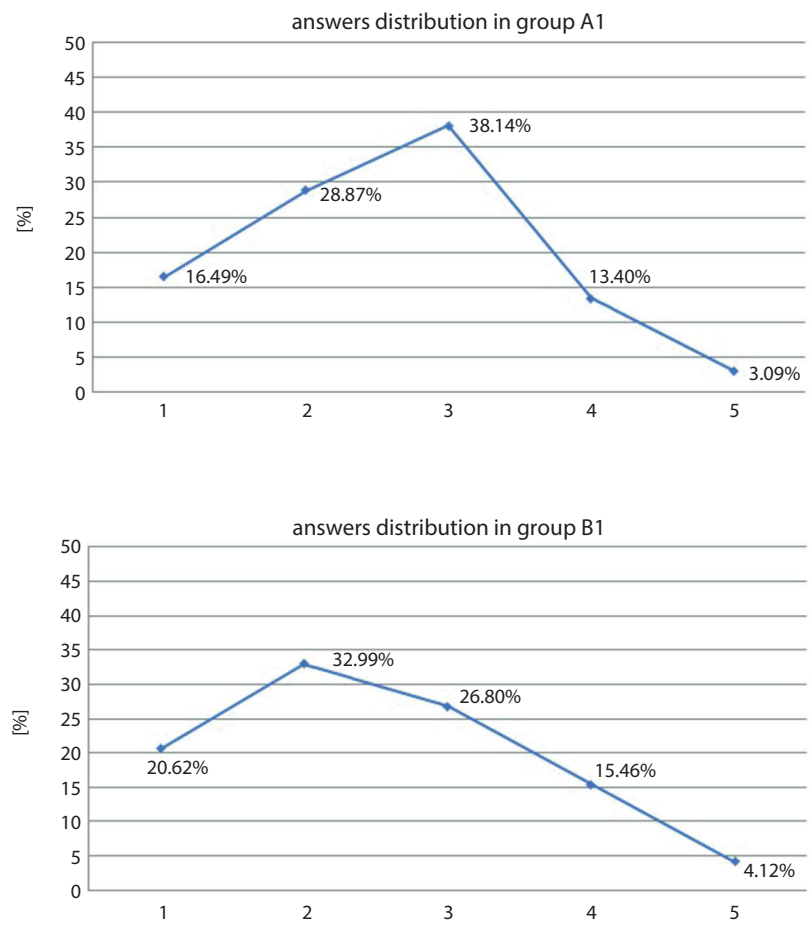

Fig. 1. Distribution of answers in each group
Table 1. Statistical assessment of comparison of the daily wear time (DWT) in patients treated with twin blocks or active plates

\begin{tabular}{|l|c|c|c|}
\hline \multirow{2}{*}{ Variable } & \multicolumn{2}{|c|}{ Type of appliance } & \multirow{2}{*}{ p-value } \\
\cline { 2 - 3 } & twin block $(\mathrm{M} \pm \mathrm{SD})$ & active plate $(\mathrm{M} \pm \mathrm{SD})$ & \\
\hline DWT [h] & $7.37 \pm 2.76$ & $7.88 \pm 3.49$ & 0.41 \\
\hline
\end{tabular}

M - median; SD - standard deviation.

Table 2. Statistical analysis of the relationship between the daily wear time (DWT) and evaluation of smile attractiveness/its importance

\begin{tabular}{|l|c|c|c|} 
Variable & R coefficient & $p$-value & $\begin{array}{c}\text { Power test analysis } \\
\text { for } a=0.05\end{array}$ \\
\hline A1 & -0.7680 & $<0.0001$ & 1.0000 \\
\hline A2 & 0.4753 & $<0.0001$ & 0.9976 \\
\hline B1 & -0.9561 & $<0.0001$ & 1.0000 \\
\hline B2 & 0.4418 & $<0.0001$ & 0.9925 \\
\hline
\end{tabular}

However, the negative correlation was twice stronger than the positive one (Table 2).

Table 3 demonstrates differentiation of the DWT depending on low (1-2 points) or high (3-5 points) scores from the questionnaires. Apparently, smile attractiveness evaluated as poor by children (A1, low) and their caregivers (B1, low) undoubtedly elongates the DWT to $9.64 \mathrm{~h}$ and $9.68 \mathrm{~h}$ respectively. So do the greater children's and the caregiver's concerns about importance of the smile attractiveness (A2, high and B2, high) - however, to a lesser extent: $7.90 \mathrm{~h}$ and $7.85 \mathrm{~h}$, respectively.

Table 4 presents comparison in pairs of the caregivers' and children's judgments. We found statistically significant correlation $(r>0.7)$ only when comparing the A1 and B1
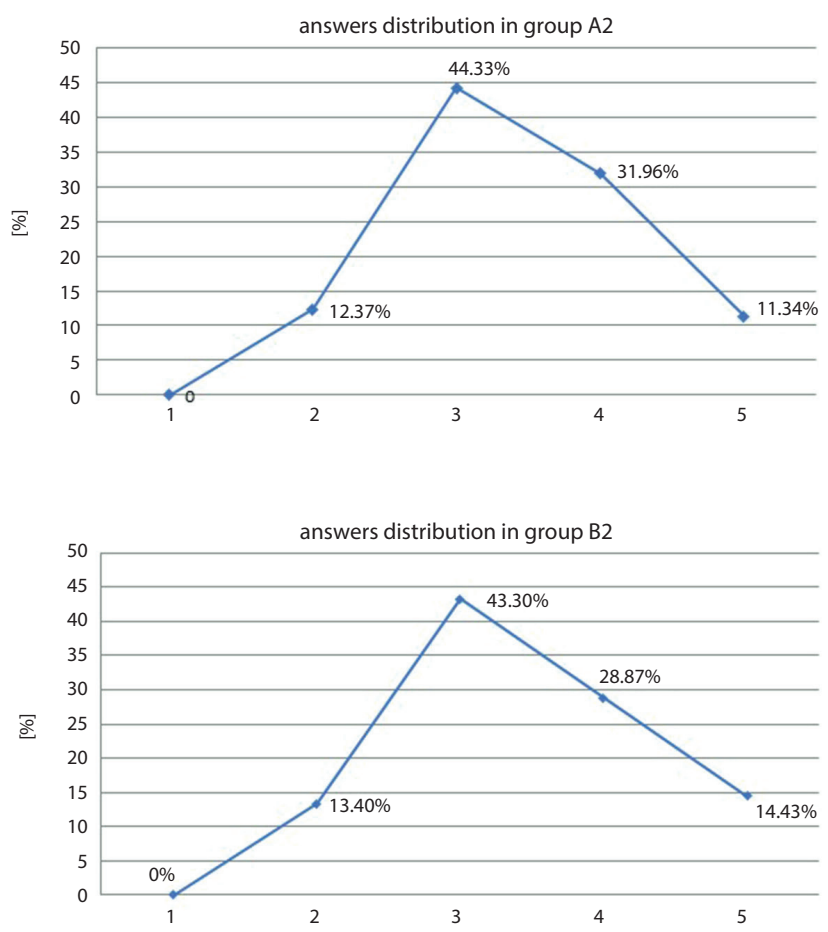
Table 3. Results of Mann-Whitney U test: intergroup comparisons between daily wear time (DWT) and subgroups

\begin{tabular}{|c|c|c|c|c|}
\hline \multirow{2}{*}{ Variable } & \multirow{2}{*}{$\begin{array}{l}\text { Admitted } \\
\text { score }\end{array}$} & \multicolumn{2}{|c|}{ DWT [h] } & \multirow{2}{*}{$p$-value } \\
\hline & & $M$ & $S D$ & \\
\hline \multirow{2}{*}{$\mathrm{A} 1$} & low & 9.64 & 2.77 & \multirow{2}{*}{0.0000} \\
\hline & high & 5.92 & 2.28 & \\
\hline \multirow{2}{*}{$\mathrm{A} 2$} & low & 5.57 & 2.68 & \multirow{2}{*}{0.0048} \\
\hline & high & 7.90 & 3.09 & \\
\hline \multirow{2}{*}{ B1 } & low & 9.68 & 2.58 & \multirow{2}{*}{0.0000} \\
\hline & high & 5.22 & 1.63 & \\
\hline \multirow{2}{*}{ B2 } & low & 6.06 & 3.11 & \multirow{2}{*}{0.0231} \\
\hline & high & 7.85 & 3.07 & \\
\hline
\end{tabular}

M - mean; SD - standard deviation.

Table 4. Statistical analysis of the variables (responses provided in the questionnaires) coherence

\begin{tabular}{|l|c|c|c|}
$\begin{array}{c}\text { Pair } \\
\text { of variables }\end{array}$ & R coefficient & p-value & $\begin{array}{c}\text { Power test analysis } \\
\text { for } \mathrm{a}=0.05\end{array}$ \\
\hline $\mathrm{A} 1$ and B1 & 0.7971 & 0.0000 & 1.0000 \\
\hline A2 and B2 & 0.8754 & 0.0000 & 1.0000 \\
\hline A1 and A2 & -0.3195 & 0.0000 & 1.0000 \\
\hline B1 and B2 & -0.4827 & 0.0000 & 1.0000 \\
\hline
\end{tabular}

or A2 and B2 variables. Interestingly, responses provided separately by the children (A1 and A 2) and their caregivers (B1 and B2) turned out to present no correlation $(r>-0.5)$.

\section{Discussion}

From a clinical point of view, it is particularly important to find a criterion that already at the stage of diagnosis would allow us to assess the future cooperation of young patients with regard to wearing their devices. And despite the fact the TheraMon ${ }^{\circledR}$ system was introduced in orthodontics a couple of years ago, such sensors have not been utilized to efficiently and reliably predict compliance instead of merely assessing it in-treatment. ${ }^{19,23,24}$ That is why our entirely new and unique approach also has a scientific long-term goal. Namely, it opens the door for the objective verification of the efficiency and efficacy of removable appliances in complying patients selected prior to manufacturing the expensive orthodontic devices. This goal, however, requires the selection of criteria that best predict the degree of cooperation among patients. The authors have already made an attempt to objectively assess the effectiveness of some of these criteria in the published studies. ${ }^{13,25}$ It is clear that conducting such a study would in the future create evidence-based treatment protocols regarding the DWT.

Sergl and Zentner ${ }^{26}$ admittedly proved that the degree of compliance depends on the orthodontic appliance type; however, in their study the DWT was not verified objectively. Our outcome (Table 1), which is in accordance with that obtained by Schafer al., ${ }^{27}$ results from monitoring patients' cooperation with sensors, thus increasing reliability of the results. Therefore, we neglected the type of removable device in subsequent statistical analyses. Since we proved that the orthodontic patients do not prefer active plates to twin blocks, we assume that a therapeutic construction bite does not hamper the patients' acceptance of the removable apparatus and - afterwards - does not impair the cooperation level. Nevertheless, the inquiry as to whether other bite jumping devices, e.g., bionators, are tolerated equally well as the twin blocks, remains unanswered.

Dissatisfaction with one's facial appearance is commonly known as the main factor contributing to a patient seeking orthodontic treatment. ${ }^{2,18,25,28,29}$ Therefore, in the literature from different decades reports can be found stating that esthetic impairment caused by malocclusion, as well as the caregivers' low rating of their child's occlusion support better cooperation during orthodontic therapy. ${ }^{30-32}$ According to Lin et al., ${ }^{29}$ even the visual presentation of post-treatment esthetics deterioration and the ensuing non-cooperation during retention phase seems to increase patients' discipline with regard to wearing their Hawley's retainers. However, assessment of the virtual compliance in the quoted papers remains in a sphere of speculation due to lack of evidence, contrary to our findings (Table 2) based on reliable, objective measurements. The strong negative correlations of the DWT and poor smile attractiveness seen by the patients $(A 1, r=-0.77$ ) or more importantly by their caregivers $(B 1, r=-0.95)$ evidently proved the role that perception of smile attractiveness plays in predicting adequate cooperation when wearing the removable appliances.

Positive, although not strong correlations of the DWT and declared children's (A2) or caregivers' (B2) attitude to the importance of a smile attractiveness (Table 2) demonstrated that the importance of smile esthetics is a more of an abstract issue than the smile esthetics itself, thus the prior cannon function as a reliable predictor of the DWT. In this way again we proved - indirectly - that aptly selected, one-question ranking self-perception of smile attractiveness is scientifically reliable as for an assessment of the young patient's future cooperation during treatment.

Orthodontic literature recommends that the wear time of removable appliances range from $12 \mathrm{~h}$ to $14 \mathrm{~h}$ per day. Regrettably, our study has proven that the discussed, objectively verified duration is less than $8 \mathrm{~h}$ on average. Furthermore, even if it reaches $10 \mathrm{~h}$ in the most cooperative patients, namely in children with a low rating of their smiles, $3 \mathrm{~h}$ of standard deviation (SD) occur (Table 3). This leaves no doubts that previous assumptions relating to treatment efficiency are overestimated, as the measured and monitored DWT were based on bias. ${ }^{33}$ They are at odds with our highly reliable outcome, which evidently 
justifies the necessity to evaluate the effectiveness of removable appliances, in order to modernize the apparently old fashioned recommendations regarding wearing time. Notwithstanding, such an assessment demands gathering only complying patients, whose simple selection is now possible, due to our presented study results.

Folger stated that parental acceptance is extremely important in orthodontic cooperation. ${ }^{34}$ Furthermore, Albino ${ }^{32}$ and Gross et al. ${ }^{35}$ also proved that due to adolescents' likely negative perceptions of orthodontic appliances, parental support and control are critical to treatment success. Our results, obtained using an objective method of patients' cooperation evaluation, fully support these findings. We demonstrated that, due to a high correlation of answers to the same question (A1 and B1: $\mathrm{r}=0.7971$; A2 and B2: $\mathrm{r}=0.8754$ ), interviewing only the caregivers as to smile attractiveness efficiently and effectively predicts the children's cooperation (Table 4). This is how, based on our previous study results, ${ }^{25}$ we are able to state that parental personality features, rather than their children's, mainly affect the future cooperation during orthodontic treatment with removable devices. Considering that neither children nor their caregivers separately are coherent in an assessment of smile attractiveness and its importance (Table 4), utilizing complex psychological questionnaires in order to predict the DWT seems to be fully justified, especially that self-perception of smile attractiveness is a reliable predictor of a young patients' compliance with an orthodontist.

As for the limitations of our study, the questionnaire we applied consisted of only 2 questions. However, such procedure of interviewing respondents is based on the data collected from the literature. ${ }^{42,45,46}$

Theoretically, the young age of the patients could also be a limiting factor. Nevertheless, numerous studies using questionnaires referencing similar issues among the peers of our patients ${ }^{35-45}$ have already proven their adequacy among patient of that age group. It has also been confirmed by a psychologist participating in related studies that the age of the respondents did not influence their understanding of the questionnaire questions and, thus, the credibility of the given answers.

The smile esthetics was subjectively assessed only prior to the initiation of orthodontic treatment. It was done on purpose, as it prevented any influence of the therapy on the discussed parameter and, therefore, its significance for the patient cooperation prognosis. It should be remembered that, along with progress in treatment, even if the motivation is initially insufficient, a health habit related to wearing orthodontic device may be developed. ${ }^{8,25}$ On the other hand, along with the improvement of smile esthetics - especially of the feature that was not initially accepted, regardless of whether it was defined or not - the commitment of the patient may decrease. Both phenomena could weaken the credibility of our result, if it had not been for the short duration of the study that made it impossible to obtain long-term effects of the treatment.

The final aspect that constitutes a limitation of the study is the fact that we have only proven the occurrence of strong correlations between the DWT and the subjective assessment of a child's smile. We have not proven, however, that there is a cause and effect relationship between these phenomena. Nonetheless, on the basis of the available literature on the subject, ${ }^{13-18,29-34}$ it is well-founded to advocate the existence of such a dependence.

\section{Conclusions}

Our study reliably proved that the patient's smile attractiveness ranked subjectively as low on a 5-point scale survey predicts the orthodontic patient's irreproachable cooperation. What is important, we have achieved the results objectively, namely by applying the TheraMon ${ }^{\circledR}$ sensors. In other words, our easy-to-use questionnaire faciltates both: changing the protocol of removable appliances wearing time during treatment and introduction of an evidence-based policy of reimbursement of such therapy from the public funds.

In order to complete our findings, there is still a need to investigate the effectiveness of removable appliances worn shorter than previously presumed, in certain malocclusions. It would also be interesting to study whether the improved smile attractiveness negatively affects the motivation to continue treatment, thereby hindering the correction of severe malocclusions that are otherwise imperceptible to the patient.

\section{ORCID iDs}

Michał Sarul (D) https://orcid.org/0000-0002-2518-0007

Joanna Antoszewska-Smith (D) https://orcid.org/0000-0001-9192-1064 Hyo-Sang Park (D) https://orcid.org/0000-0003-0227-4112

\section{References}

1. Tausche E, Luck O, Harzer W. Prevalence of malocclusions in the early mixed dentition and orthodontic treatment need. Eur JOrthod. 2004; 26(3):237-244.

2. Proffit WR, Fields HW Jr, Moray LJ. Prevalence of malocclusion and orthodontic treatment need in the United States: Estimates from the NHANES III survey. Int J Adult Orthodon Orthognath Surg. 1998; 13(2):97-106.

3. Witanowska J, Zadurska M. System solutions for orthodontic care across Europe, including world trends. Forum Ortod. 2012;8:207-214.

4. Żyła T, Kawala B, Antoszewska-Smith J, Kawala M. Black stain and dental caries: A review of the literature. Biomed Res Int. 2015;2015:469392.

5. Nimri K, Richardson A. Applicability of interceptive orthodontics in the community. British J Orthod. 1997;24(3):223-228.

6. Schott TC, Göz G. Wearing times of orthodontic devices as measured by the TheraMon ${ }^{\circledR}$ microsensor. J Orofac Orthop. 2011;72(2):103-110.

7. Stocker B, Jan H, Willmann JH, Wilmes B, Vasudavan S, Drescher D. Wear-time recording during early Class III facemask treatment using TheraMon chip technology. Am J Orthod Dentofacial Orthop. 2016; 150(3):533-540.

8. Kawala B, Antoszewska J, Sarul M, Kozanecka A. Application of microsensors to measure real wear time of removable orthodontic appliances. Czas Stomatol. 2013;66(3):321-330. 
9. Schott TC, Ludwig B. Microelectronic wear-time documentation of removable orthodontic devices detects heterogeneous wear behavior and individualizes treatment planning. Am J Orthod Dentofacial Orthop. 2014;146(2):155-160.

10. Sergl H, Zentner A. Predicting patient compliance in orthodontic treatment. Semin Orthod. 2000;6(4):231-236.

11. Albino JE, Lawrence SD, Lopes CE, Nash LB, Tedesco LA. Cooperation of adolescents in orthodontic treatment. J Behav Med. 1991;14(1): 53-70.

12. Woolass KF, Shaw WC, Viader PH, Lewis AS. The prediction of patient co-operation in orthodontic treatment. Eur J Orthod. 1988;10(3): 235-243.

13. Sarul M, Kawala B, Kozanecka A, Łyczek J, Antoszewska-Smith J. Objectively measured cooperation during early orthodontic treatment: Do the treatment needs have an impact? Adv Clin Exp Med. 2017;26(1):83-87.

14. Amado J, Sierra AM, Gallon A, Alvarez C, Baccetti T. Relationship between personality traits and cooperation of adolescent orthodontic patients. Angle Orthod. 2008;78(4):688-691.

15. Shaw WC, Rees G, Dawe M, Charles CR. The influence of dentofacial appearance on the social attractiveness of young adults. Am JOrthod. 1985;87(1):21-26.

16. de Almeida AB, Leite ICG, Melgaço CA, Marques LS. Dissatisfaction with dentofacial appearance and the normative need for orthodontic treatment: Determinant factors. Dental Press J Orthod. 2014;19(3): 120-126.

17. Giraldo LMC, Rendón ACP, Londoño AG, Vásquez JMC. Perception of adolescent patients on cooperation during orthodontic treatment: A qualitative study. Int J Odontostomat. 2014;8(2):225-228.

18. Samsonyanová L, Broukal Z. A Systematic review of individual motivational factors in orthodontic treatment: Facial attractiveness as the main motivational factor in orthodontic treatment. Int J Dent. 2014;2014:938274.

19. Pauls A, Nienkemper M, Panayotidis A, Wilmes B, Drescher D. Effects of wear time recording on the patient's compliance. Angle Orthod. 2013;83(6):1002-1008.

20. Koronacki J, Mielniczuk J. Statystyka dla kierunków technicznych iprzyrodniczych. Warszawa, Poland: WNT; 2001.

21. Colwell DJ, Gillett JR. Spearman versus Kendall. The Mathematical Gazette. 1982;66:307-309.

22. Zar JH. Biostatistical Analysis. $5^{\text {th }}$ ed. DeKalb, IL: Northern Illinois University, Pearson Education Limited; 2010.

23. Schott TC, Meyer-Gutknecht H, Mayer N, Weber J, Weimer K. A comparison between indirect and objective wear-time assessment of removable orthodontic appliances. Europ J Orthod. 2017;39(2):170-175.

24. Kirshenblatt S, Chen H, Dieltjens M, Pliska B, Almeida FR. Accuracy of thermosensitive microsensors intended to monitor patient use of removable oral appliances. J Can Dent Assoc. 2018;84:i2.

25. Sarul M, Lewandowska B, Kawala B, Kozanecka A, Antoszewska-Smith J. Objectively measured patient cooperation during early orthodontic treatment: Does psychology have an impact? Adv Clin Exp Med. 2017;26(8):1245-1251.

26. Sergl HG, Zentner A. A comparative assessment of acceptance of different types of functional appliances. Eur JOrthod. 1998;20(5):517-524.

27. Schäfer K, Ludwig B, Meyer-Gutknecht H, Schott TC. Quantifying patient adherence during active orthodontic treatment with removable appliances using microelectronic wear-time documentation. Eur J Orthod. 2015;37(1):73-80.
28. Allan TK, Hodgson EW. The use of personality measures as a determinant of patient cooperation in an orthodontic practice. Am JOrthod. 1968:54(6):433-440.

29. Lin FO, Sun H, Ni ZY, Zheng ML, Yao LJ. A feasible method to improve adherence of Hawley retainer in adolescent orthodontic patients: A randomized controlled trial. Patient Prefer Adherence. 2015;9: 1525-1530.

30. Jie Y, Dan-Dan L, Yan-Qi Y, Colman P, McGrath J, Nikos M. What are patients' expectations of orthodontic treatment: A systematic review. BMC Oral Health. 2016;16:19.

31. Dion KK, Berscheid E. Physical attractiveness and peer perception among children. Sociometry. 1974;37(1):1-12.

32. Albino JEN. Factors influencing adolescent cooperation in orthodontic treatment. Semin Orthod. 2000;6(4):214-223.

33. Chen JY, Will LA, Niederman R. Analysis of efficacy of functional appliances on mandibular growth. Am J Orthod Dentofacial Orthop. 2002;122(5):470-476

34. Folger J. Relationships of children's compliance to mothers' health beliefs and behavior. J Clin Orthod. 1988;22(7):424-426.

35. Gross AM, Samson G, Dierkes M. Patient cooperation in treatment with removable appliances: A model of patient noncompliance with treatment implications. Am J Orthod. 1985;87(5):392-397.

36. Shaw WC. The influence of children's dentofacial appearance on their social attractiveness judged by peers and lay adults. Am J Orthod. 1981;79(4):399-415.

37. Cross JF, Cross J. Age, sex, race, and the perception of facial beauty. Develop Psych. 1971;5(3):433-439.

38. Kleck R, Richardson S, Ronald L. Physical appearance cues and interpersonal attraction in children. Child Develop. 1974;45(2):305-310.

39. Lansdown R, Polak L. A study of the psychological effects of facial deformity in children. Child Care Health Dev. 1975;1(2):85-91.

40. Kjäldman I-O. Self-Concept and School Achievement of Pupils with Cleft Lip, Cleft Palate or Both. A Longitudinal Study [doctoral dissertation]. University of Helsinki, Finland; 2006.

41. Henson ST, Lindauer SJ, Gardner WG, Shroff B, Tufekci E, Best AM. Influence of dental esthetics on social perceptions of adolescents judged by peers. Am J Orthod Dentofacial Orthop. 2011;140(3):389-395.

42. Birkeland K, Bøe OE, Wisth PJ. Relationship between occlusion and satisfaction with dental appearance in orthodontically treated and untreated groups. A longitudinal study. Europ J Orthod. 2000;22(5): 509-518.

43. Planinšec J, Fošnarič S. Relationship of perceived physical self-concept and physical activity level and sex among young children. Percept Mot Skills. 2005;100(2):349-353.

44. Cavero MAB. Harter's Self-Perception Profile for Children: An adaptation and validation of the Spanish version. Psych Rep. 2014;115(2): 444-466.

45. Salih FN, Lindsten, Bågesund M. Perception of orthodontic treatment need among Swedish children, adolescents and young adults. Acta Odontologica Scandinavica. 2017;75(6):407-412.

46. Boeira GF, Salas MMS, Araújo DC, Masotti AS, Correa MB, Demarco FF. Factors influencing dental appearance satisfaction in adolescents: A cross-sectional study conducted in southern Brazil. Braz J Oral Sci. 2016;15(1):8-15. 